\title{
Taking First World Technology to Rural Schools
}

\author{
Brandon Ian Reed \\ University of Cape Town
}

\section{Introduction}

One of the core technologies that determines a country's ability to compete in the international arena is that of manufacturing and having the ability to use advanced automation tools to ensure that ones products have the highest quality to price value. In order for South Africa to compete internationally and succeed in an increasingly demanding trading arena, it is imperative that the technological awareness and ability of its population is increased. This can only be achieved by exposing and exciting its students to core technologies during their formative years.

There are very few schools in South Africa that have access to these sorts of facilities yet there is an inherent expectation that when school leavers enter the market place that they are competent in these areas. The South African Government identified Technology as one of the eight basic learning areas in its plan for their new curriculum framework, Curriculum 2005 $[1,2]$. However, in an environment of ever shrinking resources, schools are struggling to successfully accomplish this when the cost of introducing this technology is often more than their annual budget.

It is often the tertiary educational institutions that have made the significant capital investment in these facilities and they are generally situated in well-developed urban areas. Unfortunately, it is in the historically disadvantaged rural areas where there is the greatest direct need for the students to be exposed to this technology.

This paper will describe the authors work in implementing video conferencing using standard low bandwidth analogue telephone (POTS) lines to bring the advanced technology that is available in the University of Cape Town's Department of Mechanical Engineering into the classroom in a way that fosters a feeling of hands on experience of the equipment being used by the students.

\section{Effective Use Of Distance Learning For Technology Education in a Rural Environment}

In South Africa, students in rural areas have little or no access to technologies taken in most part for granted by those living in the urban areas of our country. Under these circumstances, the students are often apprehensive when exposed to sophisticated technologies. It is essential that they are not only excited by what they see in the technology, but also feel that they are empowered by what they do through hands on use of the technology under review even if it is only in a virtual environment. Allsop [3] has proposed the mnemonic PRIME (Problem Research - Ideas - Make - Evaluate) to assist these students in the uptake and understanding of the technological processes.

There is an urgent need for interaction between well-equipped tertiary institutions and schools where these institutions can share the technology that they already have, with the schools. There are two ways to accomplish this. The first is to take the students to the institutions that have the equipment, give them a talk on the technology involved and then 
demonstrate the concepts in operation. Chambers [4] suggests that students regard this form of interaction with trepidation and most end with a sense of disappointment and disillusionment as the presentations are generally pitched at too high a level for the students to understand and there is limited opportunity for them to actually do anything. Another aspect to bear in mind is the serious logistical implication in having to regularly transport students to various institutions. Issues surrounding this problem have been fully described by Kanhasuwan [5].

Holbrook [6] advocates that the process of producing something useful through the application of knowledge and skill is integral to the core idea of technology education. He goes on to emphasize the need for students to produce something in its final form rather than simply illustrating the process. Following this rationale, the second option available is to take the equipment to the schools and to let the students interact with it in their own environment. This option is however often not feasible due to the size and nature of the equipment involved.

The solution to the problem lies in a compromise between the two options. One such compromise is to make use of modern technology to allow the students to interact with machines without them actually being there. This is where the use of remote video conferencing comes into its own, allowing the students to be able to see and work with the machines in question from their own classroom environment.

In order to illustrate the ideas developed to implement this process, the use by students of a computer numerically controlled (CNC) lathe will be described. To make the experience as realistic as possible and to ensure that they are involved in the full product cycle of concept design, computer CNC code development through to final part manufacture, it was necessary to integrate the full feature set available to a CNC machine operator/designer. This includes using Computer Aided Drawing tools to initially design the part to be produced, followed by using the $\mathrm{CNC}$ code generator to produce code that controls the machining process. After the actual machining of the part, the completed part can then be inspected for amongst others, dimensional accuracy.

\section{Learning Through Hands-on Interaction}

A copy of the CAD/CAM software [7] used in the design phase of the component to be manufactured by the students was installed on the Schools computers. This is a fully functioning version of the CNC lathe software with the only difference between this and the real system is that the computer is not interfaced to the CNC lathe directly. The computers act as stand-alone design stations, one of which can later be linked via a modem to the Host computer on the CNC lathe. A carefully constructed tutorial was developed [8] that would show how the basic machining operations that they had previously done by hand on a conventional lathe could be transferred quickly and simply into an automated environment. This is considered by Aldeen [9] to be the learner-based mode of multimedia teaching where a student accesses the material directly and can receive a comprehensive treatment of the subject through a carefully constructed tutorial including simulation and self-evaluation. Heng [9] has categorized this form of interaction with the students as the objectivist model of learning. He goes on to say that in the case computer based learning, students have time to process the information at their own pace unlike the classical definition of objectivist learning where this is dictated by the instructor. 
At every step during the tutorial, the students were able to visualize the processes that they had developed and coded by running a simulation (see Figure 1) of the machining process up to that point in the software's virtual simulation environment.

It was apparent during the completion of the tutorial that some of the students had more of an affinity with the concepts involved than others. Certain assumptions about their ability to interpret the drawings in the tutorial had been made. Further

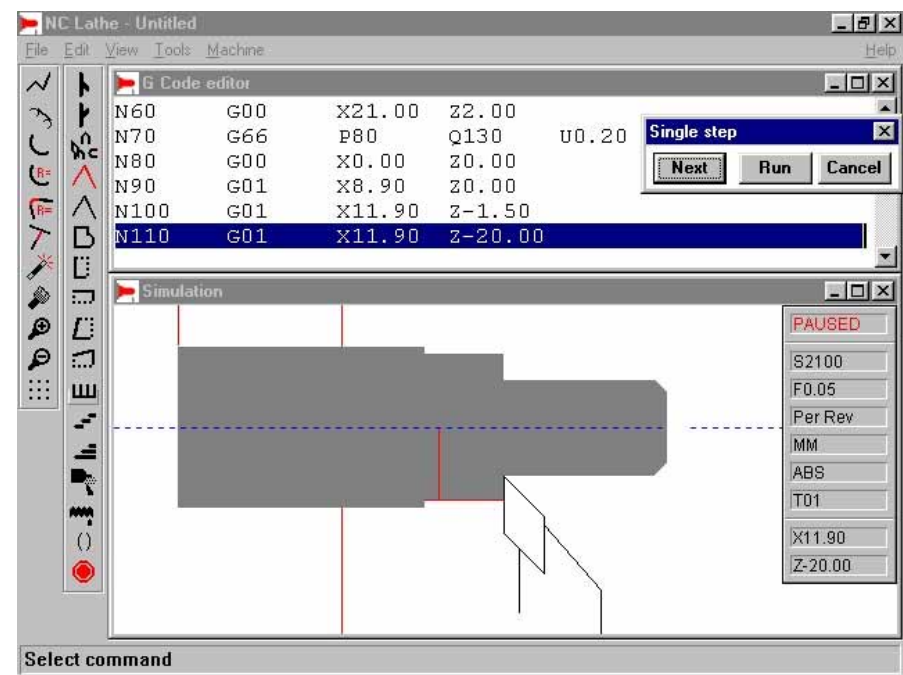

Figure 1 investigation is necessary to ensure that the tutorials are directed at the correct academic level and based on the specific abilities of the students.

By the end of the tutorial there was an air of expectation regarding the next step in the process, the actual machining. The ideal would be to have each of the students use the code that they had developed on a CNC lathe to produce the final part. This is where it is necessary to implement the compromise discussed earlier as this is often not feasible.

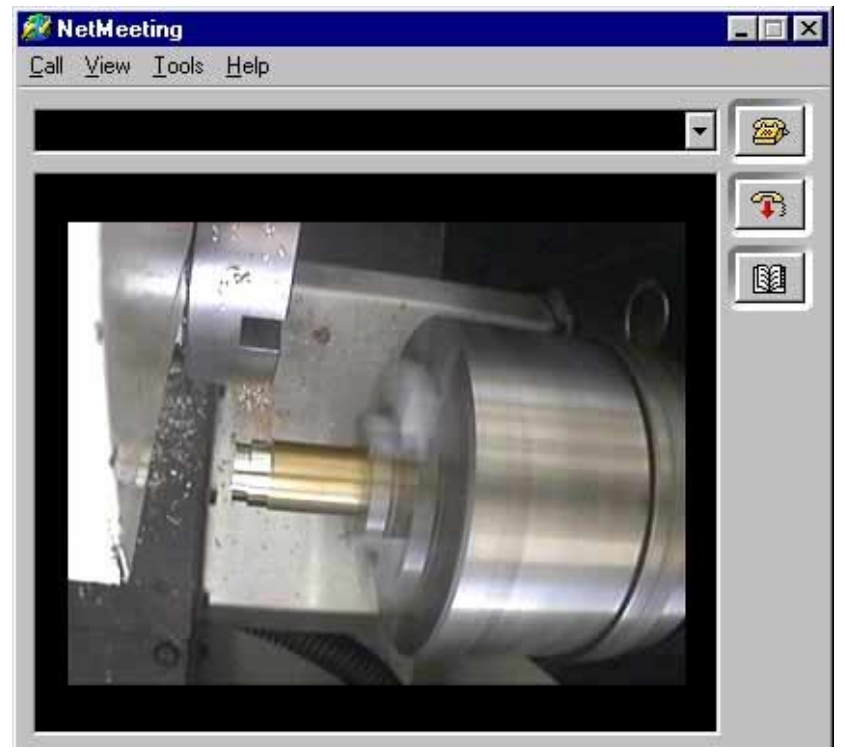

Figure 2
The solution was to use video conferencing techniques to show the students the CNC lathe in the Department's laboratory. A data projector was used to show a large screen version of the process on a wall in the classroom. CNC code generated by one of the members of the class was used to control the CNC lathe. This was done with cognisance taken of the principles put forward by Allsop [3] and Chambers [4] to ensure that the students feel that they were actually participating in the process and not just watching a demonstration. The $\mathrm{CNC}$ code was transferred to the Department using the data transfer capabilities of the video conferencing software.

Figure 2 shows one of the views of the manufacturing of the actual part in progress. Various views of the operation were shown to ensure a maximum sense of involvement by the students. To complete the learning cycle, the finished part was couriered to the school as soon as it had been machined. 


\section{Technical Requirements for the Remote Machining}

The technical requirements developed here attempts to ensure that the implementation of the first world technology requirements needed to facilitate this video transfer of information can take place even with the limited resources available in many of the schools in South Africa.

\section{a. The Host Controller}

The computer that facilitates the video conferencing environment within the manufacturing cell is a standard entry-level computer that has Windows98 Second Edition as its operating system. Part of the Dial-up Networking capabilities of this operating system is the ability to have the computer act as a dial-up server. This computer is attached to a conventional analogue telephone line via a 56kbps US Robotics modem and a low cost video-capture card and camera combination serves as the video conferencing interface. The modem and camera are integrated using version 3 of Microsoft's conferencing software called Netmeeting (partially illustrated in Figure 2).

\section{b. The Interfacing Computer}

At the school end there is only one requirement and that is a telephone line. This line is connected to the Interfacing computer (loaned to the school if necessary) using an internal $56 \mathrm{kbps}$ modem. This computer should run the same operating software as the host controller to ensure complete compatibility. Microsoft's Netmeeting completes the suite. In order to ensure maximum visualisation of the processes being presented, a data projector in a desirable extra to project the image displayed on the computer monitor onto a screen viewable by an extended group of students.

\section{c. Opening the Communication Channels}

The Interfacing computer is used to make a telephone call to the Host computer. As the Host computer has been configured as a dial-up server, it can answer this incoming call and authenticate the link between the two machines. The only protocol that is implemented is TCP/IP, the standard Internet protocol. Once the two machines are successfully communicating, the Interfacing computer initiates a collaborative Netmeeting session. At this stage, the live video feed being generated by the Host is visible on the Interfacing computer's monitor and thus on the large screen via the data projector (shown in Figure 2).

Various combinations of both picture size as well as picture quality were tested under live conditions to optimize the bandwidth limitations presented by using a single telephone connection. There was a tradeoff required between the need for the students to be able to see clearly what was happening during the machining process and there being a satisfactory refresh rate to ensure realism from the video feed.

The optimized result was to send a medium sized picture from the Host using the faster video quality setting. This medium sized picture was then digitally enlarged using a scaling factor available in Netmeeting running on the Interfacing computer.

\section{Conclusion}

This paper discusses the process whereby sophisticated computer controlled machinery can be remotely operated by students using CNC code they have developed and simple video conferencing techniques. 
There was significant interest shown by the students as to possibilities of using this $\mathrm{CAD} / \mathrm{CAM} / \mathrm{CNC}$ process in the machining of components and they expressed satisfaction and excitement about the technology that they had just seen implemented [10].

In order to cater for schools where there has been no exposure to manufacturing technology, future work would be to introduce the constructivist model of instruction as described by Heng [9] where the use of simulation and virtual reality can be used to help reinforce the link between a conventionally operated lathe and the CNC lathe tutorial that they would be required to complete.

References

1. URL: http://www.polity.org.za/govdocs/misc/curr2005.html; CURRICULUM 2005, Lifelong Learning for the $21^{\text {st }}$ Century, A User's Guide

2. Parliament of the Republic of South Africa, White Paper on Education and Training, Notice 196 of 1995 , 15 March 1995

3. R T Allsop, Factors Affecting the Uptake of Technology in Schools, in Education, Industry and Technology, ed. D J Waddington, Pergamon Press, 1987, pp 47-51

4. C Chambers, Taking the Classroom to Industry, in Education, Industry and Technology, ed. D J

Waddington, Pergamon Press, 1987, pp 191-194

5. L Kanhasuwan, An Alternative to industrial Visits: a Project in Thailand, in Education, Industry and Technology, ed. D J Waddington, Pergamon Press, 1987, pp 195-196

6. J B Holbrook, Technology Education: A union of Science and Technical Skills, in Education, Industry and Technology, ed. D J Waddington, Pergamon Press, 1987, pp 85-89

7. Software: NC Lathe, Version 1.00, Hercus CNC Machine Tools, Axmell Pty Ltd, Australia

8. Unpublished, 1999: Available on request from the Author at Mechanical Engineering, University of Cape Town, Private Bag, Rondebosch, 7701

9. LI Heng, Viewpoint: Using Information Technology to Enhance Engineering Education, International Journal of Engineering Education, Volume 13, Number 5, 1997, pp319-324

10. M Aldeen, Viewpoint: Future Directions for Multimedia-Based Engineering Education, International Journal of Engineering Education, Volume 12, Number 4, 1996, pp246-249

11. C Campbell, Rondebosch boys get a video view of the future, Cape Argus, Independent Newspapers Cape, 7 September 1999, pg 12

\section{BRANDON IAN REED}

Brandon Reed is a lecturer in the Department of Mechanical Engineering at the University of Cape Town where he is currently completing his $\mathrm{PhD}$. In addition to research, Brandon's teaching include the courses Introduction to Mechanical Engineering, which introduces students to basic engineering skills lost through the advent of computers, television and other such technologies, and Computer Integrated Manufacturing and Robotics. 